\title{
Time to Rethink
}

\section{${ }^{1}$ Dr. Suchita S. Daokar, ${ }^{2}$ Dr. Handa Amit Satish, ${ }^{3}$ Dr. Sunita Sachdeo}

Abstract: Orthodontic appliances have always been a factor impeding oral hygiene maintenance, resulting in plaque accumulation and enamel demineralization. But method of ligation as an additional factor has been evaluated by very few.

New non-conventional ligation methods are available, which reduces the friction (eg. Slide modules), but the amount of plaque accumulation with them is a concern. So, the aim of this study was to determine changes in microbial flora and periodontal status with two different archwire ligation techniques-ie conventional elastomeric modules and Slide ${ }^{\mathrm{TM}}$ (Leone Orthodontic Product).

METHOD: 10 Orthodontic patients were selected for this split-mouth study and two auxiliaries (elastomeric rings and Slide modules) for ligating archwires were tested. Microbial and periodontal records were obtained before bonding (T0), one day later (T1), and four weeks after bonding (T2). Paired t-test was applied to compare the groups statistically.

RESULT: The slide modules were associated with a higher score for plaque, bonded bracket plaque index, bleeding and greater number of microbial colonies than elastomeric modules $(P<0.05)$. Whereas no significant increase in gingival index and pocket depth was observed.

CONCLUSION: Slide modules favors plaque accumulation and it should be used cautiously in patients with poor oral hygiene.

\section{Introduction:}

Fixed or removable orthodontic appliances impede the maintenance of oral hygiene, by creating new retention areas, and ultimately resulting in plaque accumulation ${ }^{1,2,3,4}$ and bacterial colonization ${ }^{3,5}$.

The combination of orthodontic therapy and poor oral hygiene can cause serious damage to the periodontium ${ }^{6}$. Also, the plaque retention surrounding the orthodontic appliances leads to enamel demineralization caused by organic acids produced by bacteria in the dental plaque, and resulting in $\operatorname{caries}^{7,8}$. So, measures to prevent / decrease the dental biofilm accumulation must be implemented to minimize the risk for periodontal diseases in orthodontic patients.

More microorganisms were found in patients with elastomeric rings than in those with stainless steel ligature ties $^{3}$; therefore, the orthodontic ligation technique was considered as an additional factor for biofilm accumulation. ${ }^{3,9}$

Newer ligation methods have been introduced to reduce the friction between archwires and bracket slots. Significant reduction in friction has been reported for nonconventional elastomeric ligatures on conventional brackets e.g. Slide modules ${ }^{10,11}$. This system offers advantages in controlling friction, while increasing patient comfort and potentially reducing treatment time ${ }^{10}$.

So the amount of plaque accumulation with these modules was a concern and, hence the aim of this study was to determine changes in microbial flora and periodontal status with the two different archwire ligation techniques-i.e. the conventional elastomeric modules (rings) and the nonconventional elastomeric ligatures/modules (i.e. Slide ${ }^{\mathrm{TM}}$, Leone Orthodontic Products).

\section{Materials And Methods:}

A total of 10 patients scheduled for fixed orthodontic treatment were randomly selected for this splitmouth study in the age range of 15-25 years. Informed consent was obtained from all the subjects. All patients were in the permanent dentition, free of dental plaque, and with no signs of gingival inflammation before starting the study.

Patients who had taken antibiotics in the last 3 month period, poor oral hygiene, systemic diseases, pregnant women's, prior periodontal treatment in the last 3 month period were excluded from this study.

The patients were given exclusively professional guidance on oral hygiene \& Bass technique for brushing the teeth. During the 1 month study, these subjects were given no additional information about oral hygiene; and were asked to be refrain from any other oral hygiene products for the duration of the trial.

At baseline, before orthodontic appliance placement, biofilm samples were collected from the labial surfaces of the maxillary second premolars and the mandibular lateral incisors (4 samples per patient). The clinical parameters recorded included Gingival index $(\mathrm{GI})^{12}$, Gingival sulcus bleeding index ${ }^{14}$,Probing depth(PD) measured by a Williams periodontal probe and the Bonded bracket plaque index(BBPI $)^{13}$. 
After the clinical examinations, the patients received fixed orthodontic appliances. Standard metal brackets $(0.022$ " MBT Prescription) were used in all patients, and 0.014 " Nitinol archwire was engaged in the appliance immediately after bonding for initial aligning and leveling.

In all patients, conventional elastomeric rings were used in maxilla and mandible for ligation on one side of the dental arch midline, with Slide ${ }^{\mathrm{TM}}$ modules (Leone orthodontic Product) on the opposite side (figures 1 and 2). Each patient's side receiving slide modules was randomly decided; so minimizing the individual preference of tooth brushing side.

Microbial and periodontal records were obtained before bonding (T0), one day after bonding (T1), and four weeks after bonding (T2).

Clinical parameters were recorded in 6 sites (mesiobuccal, buccal, distobuccal, mesiolingual, lingual, and distolingual) of all teeth (figure 3), except the molars. Only 1 professional, blinded to the groups, made all clinical parameter evaluations when the biofilm samples were collected.

For the collection of biofilm, the areas were isolated with sterile cotton rolls to prevent contamination by saliva. The elastomeric rings and Slide modules were removed and the plaque accumulation on it was transferred to the Blood agar plates. The samples were cultivated and analyzed by the same examiner in the Department of Clinical Microbiology. The plates were incubated in the incubation chamber for 24 hours and then the microbial colonies were examined for conformation regarding its type.

\section{Statistical Analysis:}

The mean and standard deviations of the gingival index, Gingival sulcus bleeding index, Probing Depth and BBPI values were calculated for both groups.

Paired $t$-test was applied to compare all parameters of the groups statistically.

To determine the longitudinal changes that occurred in the periodontal status, GI, gingival sulcus bleeding index, BBPI, and PD values of both groups were pooled.

A $P$ value of 0.05 was considered significant.

TABLE:1

\begin{tabular}{|c|c|c|c|c|c|c|c|c|c|c|c|c|c|c|c|}
\hline & \multicolumn{5}{|c|}{ T0 } & \multicolumn{5}{|c|}{ T1 } & \multicolumn{5}{|c|}{ T2 } \\
\hline & \multicolumn{2}{|c|}{ SLIDE } & \multicolumn{3}{|c|}{ ELASTOMER } & \multicolumn{2}{|c|}{ SLIDE } & \multicolumn{3}{|c|}{ ELASTOMER } & \multicolumn{2}{|c|}{ SLIDE } & \multicolumn{3}{|c|}{ ELASTOMER } \\
\hline & $\begin{array}{l}\text { ME } \\
\text { AN }\end{array}$ & S.D & $\begin{array}{l}\text { ME } \\
\text { AN }\end{array}$ & S.D & $\mathrm{P}$ & $\begin{array}{l}\text { ME } \\
\text { AN }\end{array}$ & S.D & $\begin{array}{l}\text { ME } \\
\text { AN }\end{array}$ & S.D & $\mathrm{P}$ & $\begin{array}{l}\text { ME } \\
\text { AN }\end{array}$ & S.D & $\begin{array}{l}\text { ME } \\
\text { AN }\end{array}$ & S.D & $\mathrm{P}$ \\
\hline $\begin{array}{l}\text { Gingival } \\
\text { index }\end{array}$ & 0.33 & 0.40 & 0.34 & 0.46 & NS & 0.30 & 0.50 & 0.36 & 0.45 & NS & 1.0 & 0.66 & 0.60 & 0.58 & NS \\
\hline $\begin{array}{l}\text { Bonded } \\
\text { bracket } \\
\text { plaque } \\
\text { index }\end{array}$ & 0.17 & 0.20 & 0.15 & 0.23 & NS & 0.50 & 0.51 & 0.31 & 0.42 & NS & 1.1 & 0.75 & 0.70 & 0.45 & $* *$ \\
\hline$\frac{\text { Pocket }}{\text { depth }}$ & 1.40 & 0.36 & 1.40 & 0.38 & NS & 1.42 & 0.38 & 1.38 & 0.40 & NS & 1.45 & 0.41 & 1.42 & 0.45 & NS \\
\hline $\begin{array}{l}\text { Ging.sul } \\
\text { cus } \\
\text { bleeding } \\
\text { index }\end{array}$ & 0.32 & 0.48 & 0.35 & 0.46 & NS & 0.50 & 0.46 & 0.48 & 0.51 & NS & 0.98 & 0.61 & 0.59 & 0.63 & $* *$ \\
\hline
\end{tabular}

NS indicates nonsignificant. And $* * \mathrm{P}<0.01, * * * \mathrm{P}<0.001$

\section{Gingival Index (GI):-}

\section{Results:}

No significant difference was found at any time point between the GI values of the groups (Table 1). A statistically significant increase in the GI was determined between T0 and T2 $(\mathrm{P}<0.01)$.

\section{Bonded bracket plaque index (BBPI):-}

Significant difference was found between the BBPI values of the groups at T2 (Table 1). A statistically significant increase in BBPI values was determined between T0-T1 and T0-T2 (P <0 .001) (figure 4 and 5).

\section{Pocket depth (PD):-}

No significant difference was found at any time point between the PD values of the groups (Table 1).No statistically significant difference in PD values was determined between TO-T1 and T0-T2. 
Bleeding on probing (BOP):-

In T0 and T1, the number of teeth that exhibited BOP was quite similar for both groups. However, in $\mathrm{T} 2$, significantly more teeth exhibited bleeding in the slide module group (Table 1), and results were statistically significant (figure 6 and 7).

\section{Amount of microbial colonization :--}

The amount of bacterial colonies was almost similar at the baseline. But the microbial colonies count was higher in the Slide module group compared to elastomeric group at T2.

\section{Discussion:}

There is a clear demonstration in the literature that fixed orthodontic appliances increase plaque accumulation, bacterial colonization, and resultant enamel decalcification ${ }^{6,8,15,16}$.

But, the role of ligation materials has only been evaluated in a few studies ${ }^{3,5,17}$

Our methodology of cross-arch study for microbiologic evaluation and periodontal status, of teeth with elastomeric rings and slide module comparison, was a condition to reduce individual variations and intragroup differences.

In our study, upper second pre-molars were selected as donor site because posterior teeth are more prone for plaque accumulation and lateral incisor because they are more prone to plaque and white spot lesions.

Bacterial sampling was performed before bonding (T0), one day later (T1),and four weeks after bonding (T2).The third occasion (T2) was performed four weeks after bonding, which is equivalent to the average duration between orthodontic appointments.

Forsberg et $\mathrm{al}^{5}$ evaluated microbial colonization of 12 patients treated by fixed orthodontic appliances and reported that the lateral incisor attached to the archwire with an elastomeric ring exhibited a greater number of microorganisms in the plaque than teeth ligated with steel wire.

Whereas, Sukontapatipark et $\mathrm{al}^{17}$ using scanning electron microscopy reported that the method of ligation did not appear to influence the bacterial morphotypes on either composite or enamel surfaces. But in this study, the archwire was not ligated as the study had only one bonded tooth per quadrant.

But the amount of plaque accumulation and periodontal status with non-conventional modules, ie Slide, is also a concern.

Our study revealed that teeth ligated with Slide modules exhibited more amount of plaque than teeth ligated with elastomeric rings (figure 4 and 5), and so were the bacterial colonies which were confirmed by microbial studies.

In our study, although the Gingival Index(GI) was more in slide group, but was not statistically significant, when compared among groups. Whereas, it revealed a significant increase in GI value between T0 and T2, but the final GI values were still within the boundaries of mild inflammation.

BBPI values of bonded teeth increased significantly between the groups and between T0-T1 and T0T2.The increase is in agreement with the results of some studies that suggest of change in plaque volume with change in method of ligation ${ }^{6,17,18}$ (figure 4 and 5). In our study, the slide modules morphology and the tube-like effect created by the modules may have favored more plaque accumulation around the brackets, than conventional elastomeric rings. Also it was seen that the BBPI scores were higher in posterior teeth than in anterior.

In the present study, no significant difference in Pocket Depths of bonded teeth was determined. Huser et $\mathrm{al}^{6}$ also reported that probing depth remained within normal values when method of ligation was changed.

In our study, significant differences in bleeding scores were detected. Teeth ligated with elastomeric rings exhibited less bleeding than teeth ligated with slide modules (figure 6 and 7). One feasible explanation is that bleeding may be induced by the quality and the quantity of plaque around slide modules; which favoured more plaque accumulation. The differences in surface characteristics / shape of slide modules may also be a factor.

\section{Conclusion}

- Teeth ligated with slide modules exhibited a greater amount of plaque and microbial colonies than teeth ligated with conventional elastomeric modules.

- Significant retention of plaque by Slide modules altered the Bonded Bracket Plaque Index and Gingival sulcus bleeding index.

- Therefore, the use of slide modules is not recommended in patients with poor oral hygiene, and periodontally compromised patient's.

- Also as more plaque accumulation was seen in posteriors than in the anterior teeth; the use of Slide should be avoided in posteriors and patients should be continuously reinforced for oral hygiene maintenance. 


\section{References:}

[1] Batoni G, Pardini M, Giannotti A, Ota F, Giuca MR, Gabriele M,Campa M, Senesi S. Effect of removable orthodontic appliances on oral colonization by mutans streptococci in children. Eur J Oral Sci. 2001;109:388-392.

[2] Jordan C, LeBlanc DJ. Influences of orthodontic appliances on oral populations of mutans streptococci. Oral Microbiol Immunol. 2002;17:65-71.

[3] Hakan Tu“rkkahraman,M. O“ zgu“ r Sayın; F. Yes, im Bozkurt; Zuhal Yetkin; Selc, uk Kaya, Su“leyman O` nal..Archwire Ligation Techniques, Microbial Colonization, and Periodontal Status in Orthodontically Treated Patients. (Angle Orthod 2005;75:231-236.)

[4] Kloehn JS, Pfeifer JS. The effect of orthodontic treatment on the periodontium. Angle Orthod 1974;44:127-34.

[5] Forsberg CM, Brattstrom V, Malmberg E, Nord CE. Ligature wires and elastomeric rings:two methods of ligation, and their association with microbial colonization of Streptococcus mutans and lactobacilli. Eur J Orthod 1991;13:416-20.

[6] Huser MC, Baehni PC, Lang R. Effects of orthodontic bands on microbiologic and clinical parameters. Am J Orthod Dentofacial Orthop 1990;97:213-8.

[7] Arends J, Christofferson I. The nature of early caries lesions in enamel. J Dent Res. 1986;65:2-11.

[8] O'Reilly MM, Featherstone JD. Demineralization and remineralization around orthodontic appliances: an in vivo study. Am $J$ Orthod Dentofacial Orthop. 1987;92:33-40.

[9] Ricardo Alves de Souza,a Maria Beatriz Borges de Araújo Magnani.;Periodontal and microbiologic evaluation of 2 methods of archwire ligation: Ligature wires and elastomeric rings; Am J Orthod Dentofacial Orthop 2008;134:506-12

[10] Arturo Fortini,Massimo Lupoli ;Vittorio Cacciafesta .-A New Low-Friction Ligation System; JCO/AUGUST 2005.

[11] Kapila, S.; Angolkar, P.V.; Duncanson, M.G.; and Nanda, R.S.:Evaluation of friction between edgewise stainless steel brackets and orthodontic wires of four alloys, Am. J. Orthod. 98:117- 126, 1990.

[12] Loe H. The gingival index, the plaque index and the retention index systems. J Periodontol. 1967;38(suppl):610-616.

[13] Hannah JJ, Johnson JD, Kuftinec MM. Long term clinical evaluation of toothpaste and oral rinse containing sanguinaria extract in controlling plaque, gingival inflammation and sulcus bleeding during orthodontic treatment. Am J Orthod Dentofacial Orthop. 1989;96:199-207.

[14] Greenstein G. The role of bleeding upon probing in the diagnosis of periodontal disease. A literature review. J Periodontol. 1984; 55:684-688.

[15] Scheie AA, Arneberg P, Krogstad O. Effect of orthodontic treatment on prevalence of Streptococcus mutans in plaque and saliva. Scand J Dent Res. 1984;92:211-217.

[16] Sinclair PM, Berry CW, Bennett CL, Israelson H. Changes in gingiva and gingival flora with bonding and banding. Angle Orthod. 1987;57:271-278

[17] Sukontapatipark W, el-Agroudi MA, Selliseth NJ, Thunold K, Selvig KA. Bacterial colonization associated with fixed orthodontic appliances. A scanning electron microscopy study. Eur J Orthod.2001;23:475-484.

[18] Chang HS, Walsh LJ, Freer TJ. The effect of orthodontic treatment on salivary flow, pH, buffer capacity, and levels of mutans streptococci and lactobacilli. Aust Orthod J. 1999;15:229-234.

[19] Glans R, Larsson E, Ogaard B. Longitudinal changes in gingival condition in crowded and noncrowded dentitions subjected to fixed orthodontic treatment. Am J Orthod Dentofacial Orthop.2003;124:679-682.

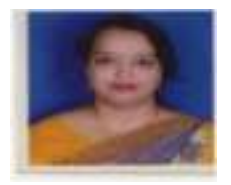

Dr. Suchita S. Daokar, Professor and P.G. Guide, Department of Orthodontics and Dentofacial Orthopaedics, C.S.M.S.S. Dental College and hospital, Kanchanwadi, Aurangabad, Maharashtra, India.

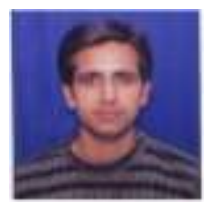

Dr. Handa Amit Satish, Post-Graduate student, Department of Orthodontics and Dentofacial Orthopaedics, C.S.M.S.S. Dental College and hospital, Kanchanwadi, Aurangabad, Maharashtra, India.

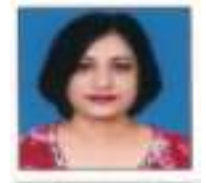

Dr. Sunita Sachdeo, Professor, Department of Microbiology, C.S.M.S.S. Dental College and hospital, Kanchanwadi, Aurangabad, Maharashtra, India.

FIGURES:--

Fig 1:-after bonding conventional module side

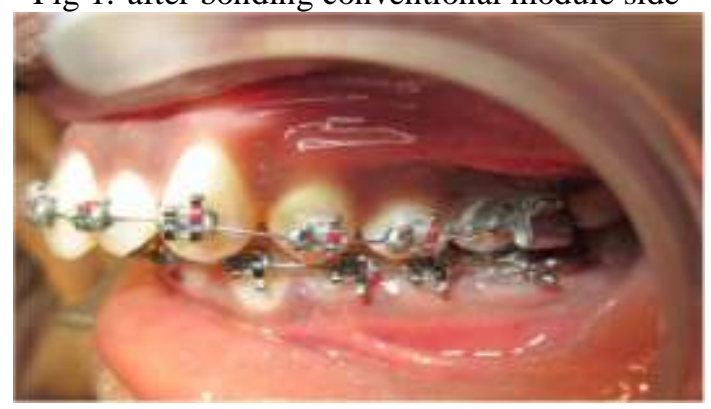


Fig 2:-after bonding with slide modules

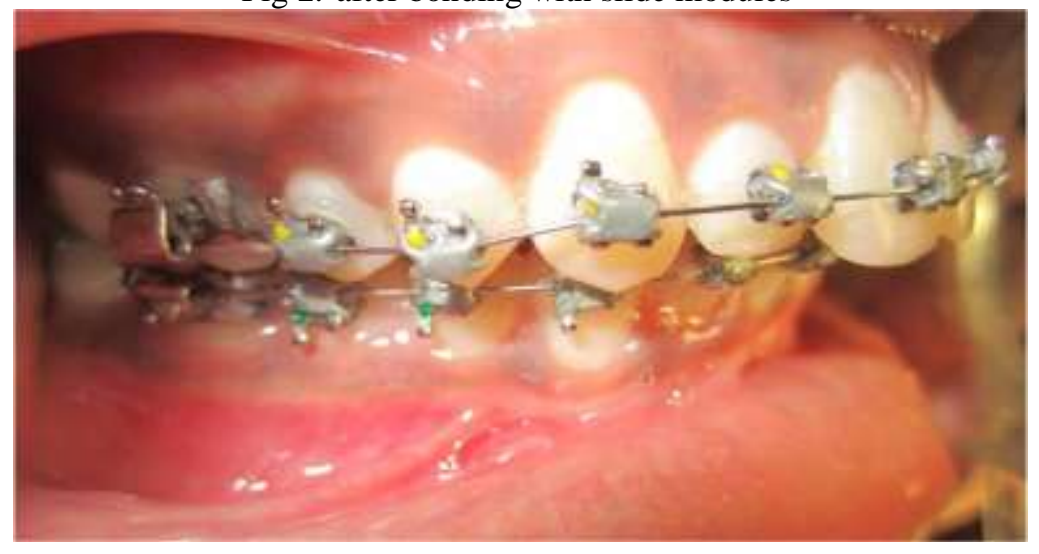

Fig 3:- clinical parameter recording

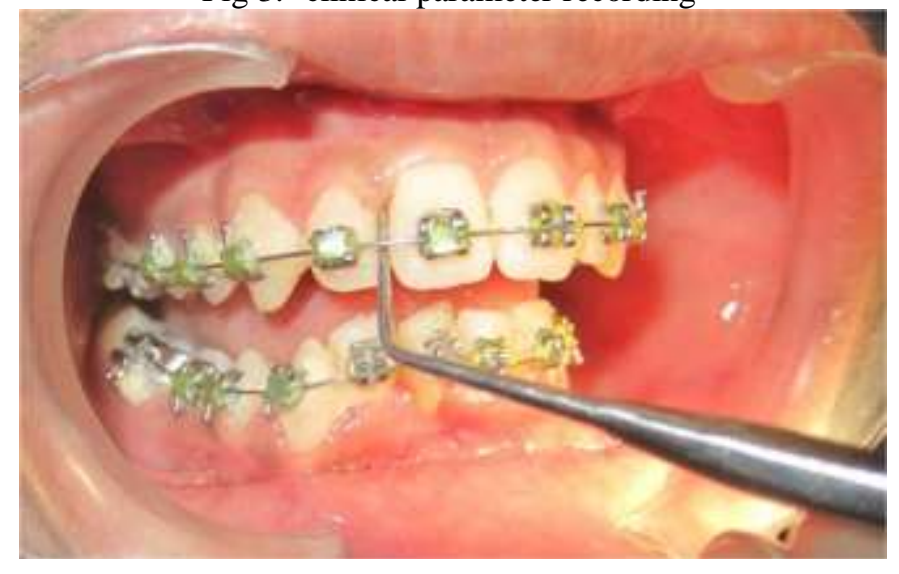

Fig 4:-decreased plaque and BBPI on conventional module side

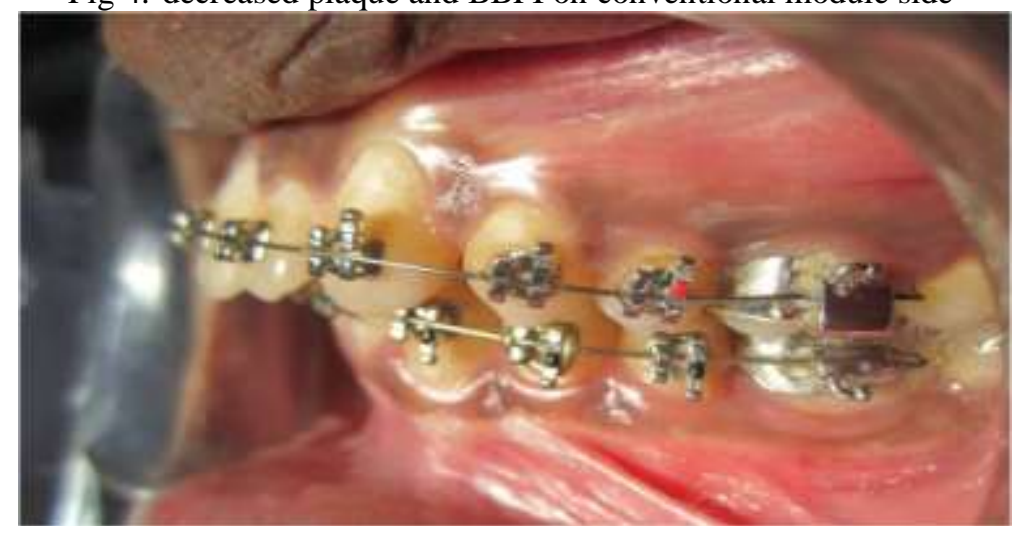

Fig 5:- increased plaque and BBPI on slide side

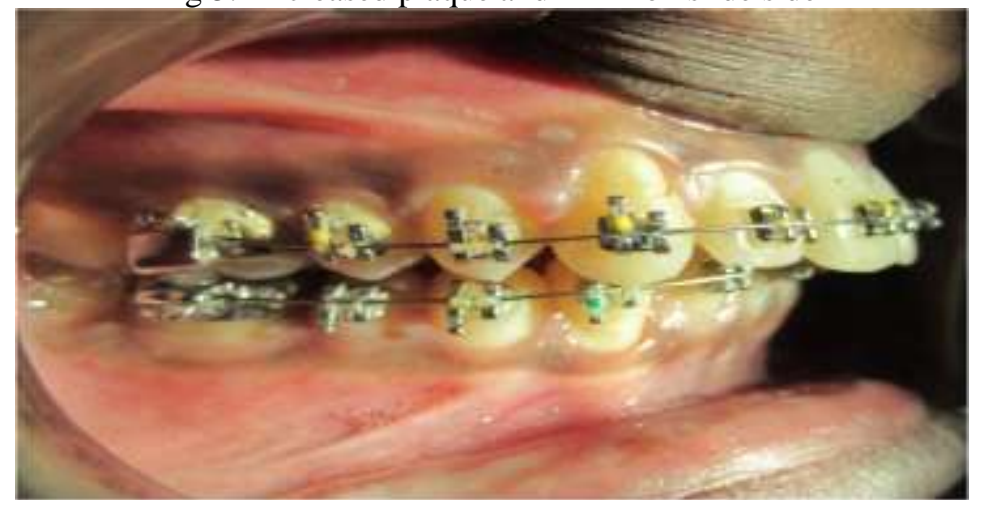


Fig.6-lesser bleeding on probing on conventional module side

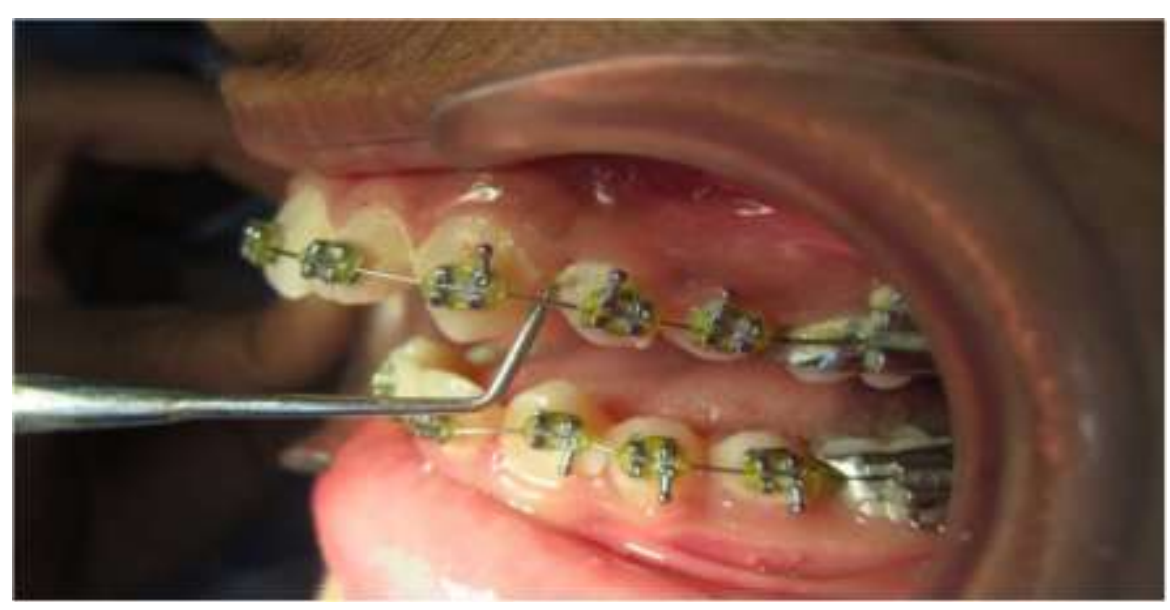

Fig.7-increased bleeding on slide module side

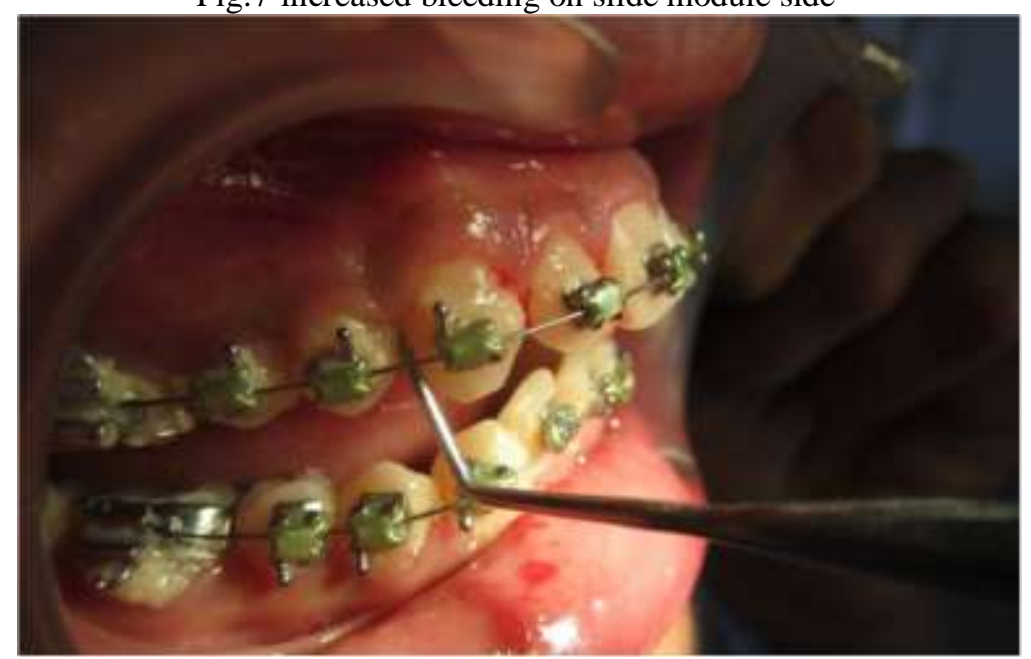

